\title{
Magneto-chiral anisotropy in charge transport through single-walled carbon nanotubes
}

\author{
V. Krstića),b) \\ Grenoble High Magnetic Field Laboratory, Max Planck Institut für Festkörperforschung/CNRS, \\ BP 166, F-38042 Grenoble, France and Max Planck Institut für Festkörperforschung, Heisenbergstraße 1, \\ D-70569 Stuttgart, Germany
}

S. Roth, M. Burghard, and K. Kern

Max Planck Institut für Festkörperforschung, Heisenbergstraße 1, D-70569 Stuttgart, Germany

\begin{abstract}
G. L. J. A. Rikken ${ }^{\text {a),c) }}$
Grenoble High Magnetic Field Laboratory, Max Planck Institut für Festkörperforschung/CNRS, BP 166, F-38042 Grenoble, France and Laboratoire National des Champs Magnétiques Pulsés, CNRS/INSA/UPS, UMS 5462, BP 4245, 31432 Toulouse Cedex, France
\end{abstract}

(Received 28 June 2002; accepted 30 September 2002)

\begin{abstract}
Carbon nanotubes are chiral molecular objects and therefore exist in two forms that are each other's mirror image. Many aspects of these fascinating new materials have recently been explored but their chirality has hardly been addressed. We have investigated the charge transport through individual single-walled carbon nanotubes in the presence of a magnetic field parallel to the tube axis. For most of the investigated tubes, a dependence of the resistance that is odd in both the magnetic field and in the current through the tube is observed. This effect is ascribed to the chirality of the nanotube and is called electrical magneto-chiral anisotropy. (c) 2002 American Institute of Physics.
\end{abstract}

[DOI: $10.1063 / 1.1523895]$

The electrical properties of single- and multiwalled carbon nanotubes (SWNTs and MWNTs) have been intensely investigated recently. Principally, carbon nanotubes (CNTs) can be thought of as a graphene sheet ${ }^{1}$ of some microns in length and a few nanometers in width that has been rolled up seamlessly. Thus CNTs exhibit a large aspect ratio and therefore have a one-dimensional character as is confirmed by the observation of van Hove singularities in the electronic density of states ${ }^{2,3}$ or the phonon spectrum. ${ }^{4}$ Different wrapping angles are possible and determine the diameter and whether a CNT is metallic or semiconducting. ${ }^{5}$ This leads to a variety of CNTs, ${ }^{5}$ classified by the pair of indices $(n, m)$. The wrapping can occur in two possible directions, leading to left- $(L)$ and right- $(D)$ handed chiral nanotubes. Among the various classes of nanotubes, there are only two special ones that are not chiral, the so-called zig-zag $(m=0)$ and armchair $(n$ $=m$ ) tubes. ${ }^{5}$ Raman scattering and scanning tunneling microscopy (STM) has been used to address the chiral structure of nanotubes. While STM can determine the molecular structure only on conducting substrates, ${ }^{2,3,7}$ Raman scattering provides no information on the handedness. ${ }^{6}$

In the charge transport effects in nanotubes investigated so far, e.g., Coulomb-blockade, ${ }^{8,9}$ signatures of Luttingerliquid behavior, ${ }^{10}$ one-dimensional localization in rings of CNTs, ${ }^{11}$ Aharonov-Bohm effect, ${ }^{12}$ and ballistic transport, ${ }^{13,14}$ chiral aspects of CNTs were never reported, although there have been calculations that suggest that the chirality should affect transport properties. ${ }^{15-17}$

\footnotetext{
a) Authors to whom correspondence should be addressed.

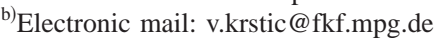

${ }^{c}$ Electronic mail: rikken@polycnrs-gre.fr
}

Recently Rikken et al. ${ }^{18,19}$ have observed a new magneto-optical phenomenon in the luminescence/ absorption spectra of chiral media, called magneto-chiral anisotropy (MChA), which depends on the wave vector $\mathbf{k}$ of the light and the magnetic field $\mathbf{B}$. The essential features of MChA are (i) its linear dependence on $\mathbf{k} \cdot \mathbf{B}$, (ii) its dependence on the handedness of the chiral medium (enantioselectivity), and (iii) its independence of the polarization state of the light. In view of this latter aspect and the theoretical work mentioned above, the question comes to mind if an analogous effect exists for electronic transport in molecular conductors, which could be used to study chirality in CNTs. The present work investigates the charge transport in SWNTs in the presence of a magnetic field in order to answer this question.

As in the optical case, the existence of MChA in chiral electrical conductors can be deduced from symmetry arguments. First we consider ballistic transport. Time- and parityreversal symmetry arguments impose that up to the first and second order magnetic corrections, the electrical resistance is given by

$$
R^{D / L}(\mathbf{I}, \mathbf{B})=R_{0}\left\{1+\beta B^{2}+\chi^{D / L} \mathbf{I} \cdot \mathbf{B}\right\},
$$

where $\mathbf{I}$ is the current traversing the conductor and $\mathbf{B}$ is the external magnetic field. The parameters $\chi^{D}=-\chi^{L}$ denote the handedness of the chiral conductor. The parameter $\beta$ describes the normal longitudinal magnetoresistance that is allowed in all conductors. We call the effect corresponding to the last term on the right-hand side of Eq. (1) electrical magneto-chiral anisotropy (eMChA). 
In the case of macroscopic diffusive transport, one has to use the Onsager relation (for a discussion see, e.g., Ref. 20), which states that under time-reversal the conductivity tensor elements $\sigma_{k l}$ must behave as

$$
\sigma_{k l}(\mathbf{j}, \mathbf{B})=\sigma_{l k}(-\mathbf{j},-\mathbf{B})
$$

with $\mathbf{j}$ the current density and $\mathbf{B}$ the magnetic field. This relation must also hold for the resistivity tensor elements $\rho_{k l}(\mathbf{j}, \mathbf{B}) \equiv(\sigma(\mathbf{j}, \mathbf{B}))_{k l}^{-1}$. Expanding $\rho_{k l}(\mathbf{j}, \mathbf{B})$ under consideration of Onsager's relation and imposing parity symmetry one obtains, up to second order terms,

$$
\left.\rho^{D / L}(\mathbf{j}, \mathbf{B})=\rho_{0}\left\{1+\alpha \mathbf{j}^{2}+\beta B^{2}+\chi^{D / L} \mathbf{j} \cdot \mathbf{B}\right)\right\},
$$

which is equivalent to Eq. (1) as far as eMChA is concerned. Therefore for both cases, symmetry considerations show that eMChA is an allowed effect in chiral conductors. Note that also in both cases higher order odd terms of the form $\mathbf{j}^{2 p+1} \mathbf{B}^{2 q+1}$ ( $p, q$ integer) are allowed, but were omitted from the equations for simplicity. Recent experiments on macroscopic chiral conductors have confirmed the validity of Eq. (3). ${ }^{21}$ For carbon nanotubes the odd power dependence on the magnetic field and the current was discovered in the theoretical works of Spivak et al. ${ }^{17}$ and Kibis. ${ }^{16}$

Our measurements of $\chi^{D / L}$ have been carried out on two-terminal contacted SWNT bundles with the magnetic field $B$ along the bundle axis (Fig. 1, Ref. 23). Only SWNTs with room-temperature resistances between 7.1 and $12.8 \mathrm{k} \Omega$, have been investigated. This implies both a small contact resistance, and a metallic character of the tube. Indeed, in these SWNT, the resistance values did not change by more than $10 \%$ upon cooling to $4.2 \mathrm{~K}$. The resistance anisotropy $\delta R(B, I) \equiv R(B, I)-R(B,-I)$ is determined by standard lock-in techniques. Due to unintentional, small differences between the two contacts to the tube, this quantity can have a contact resistance anisotropy contribution. Such a contribution can however only have an even magnetic field dependence and to eliminate it, we determine the resistance anisotropy difference,

$$
\Delta R(B, I) \equiv \delta R(B, I)-\delta R(-B, I)=4 \chi^{D / L} B I .
$$

In Fig. 2(a) the $\Delta R(B, I)$ of the nanotube in Fig. 1 is plotted as a function of the absolute value of the magnetic field. In the inset $\delta R(B, I)$ is shown. From both it is obvious that the term linear in $B$ is clearly dominant and only a small contribution from the terms proportional to $B^{2}$ is apparent, which we ascribe to contact artefacts. Such behavior was observed for several tubes. From Fig. 2(a), the absolute value of $\chi^{D / L}$ can be estimated to be about $500 \mathrm{k} \Omega / \mathrm{AT}$. We define $\eta \equiv \lim _{B \rightarrow 0} \partial \Delta R / \partial B$ as the eMChA slope, which in this sample yields $\eta>0$. At a given current and field orientation, the two signs of $\eta$ are assigned to the two handednesses of the SWNTs. At this time, it is not possible to determine which sign corresponds to which handedness. Either an independent experimental identification of the handedness of the tubes, or a rigorous theoretical assignment is required. It should be noted that the clear observation of eMChA proves that charge transport through CNTs is sensitive to chirality, and therefore must have a three-dimensional character.
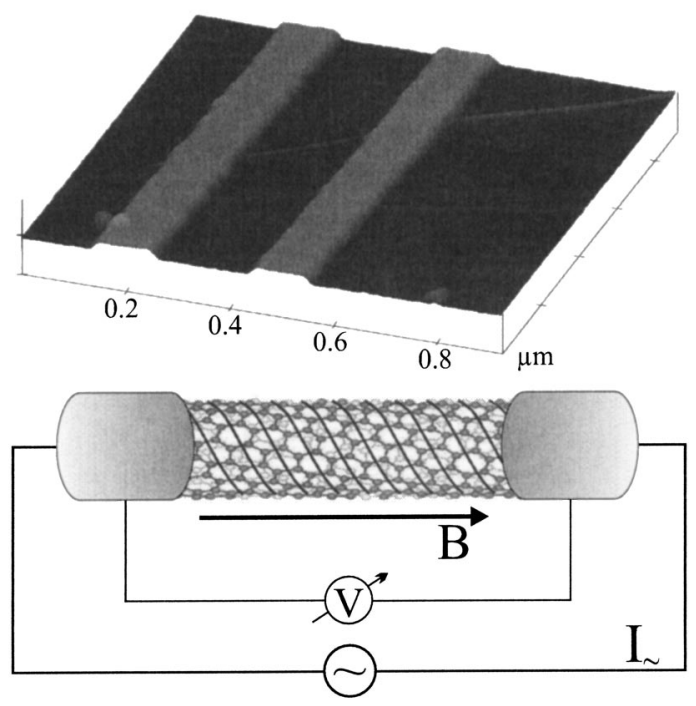

FIG. 1. Experimental details and set-up. Shown is an AFM image of a typical nanotube sample and an illustration of a $(10,5)$ nanotube connected in two-point configuration to an ac current source. In the latter the chiral character of the nanotube is highlighted by the solid lines. The external magnetic field $B$ is applied along the SWNT symmetry axis to within a few degrees. The AuPd electrodes are $16 \mathrm{~nm}$ in height, $125 \mathrm{~nm}$ in width, and 195 $\mathrm{nm}$ apart. The height of the thin nanotube bundle is about $1.8 \mathrm{~nm}$. For sample preparation, see Refs. 22 and 14. The measurement electronics is built around a $V / I$-converter and two lock-in amplifiers. The $V / I$-converter adds the output oscillator-voltages of the lock-ins and converts this sum into an output current. This current which is sent through the SWNT consists of a small amplitude high frequency part and a large amplitude low frequency part. Phase sensitive detection of the high frequency part yields the resistance, low frequency phase sensitive detection on this resistance yields the resistance anisotropy. By replacing the SWNT by a standard $10 \mathrm{k} \Omega$ resistor and for $I=500 \mathrm{nA}$, the anisotropy resolution of the electronics was found to be $\leqslant 5 \mathrm{~m} \Omega$. Noise and drift on the anisotropy of SWNT varied between 0.1 $\Omega$ and $1 \Omega$, depending on contact quality.

Figure 2(b) shows $\delta R(B, I)$ (inset) and $\Delta R(B, I)$ up to 5 $\mathrm{T}$ for another SWNT. At higher magnetic fields (not shown for clarity) additional structure is observed, which probably originates from some resonant contributions to the eMChA which are not taken into account in the above series expansion of the resistance [Eq. (3)]. However, these contributions are beyond the focus of the present discussion and will be addressed elsewhere. The behavior of $\delta R(B, I)$ in the inset of Fig. 2(b) shows a dominating quadratic $B$ term. This quadratic term is much larger than in Fig. 2(a), suggesting that the tube of Fig. 2(b) contains more scattering centres and/or a higher contact resistance anisotropy contribution. Evaluating $\Delta R(B, I)[$ main Fig. 2(b)] from the data, a negative eMChA slope, i.e., $\eta<0$ is apparent and $\chi^{D / L}$ can be approximated to be $-934 \mathrm{k} \Omega / \mathrm{AT}$ for this particular tube. This value for the parameter $\chi^{D / L}$ indicates that this tube has a different structure, i.e., another pair of integers $(n, m)$, than the tube in Fig. 1.

In Fig. 2(c) a histogram on the occurrence of the relative handedness of all investigated SWNT samples is shown. Five SWNTs with $\eta<0$, four SWNTs with $\eta>0$, and only one with $\eta=0$ were found during our measurements. The tube with $\eta=0$ has to be either an armchair or a zig-zag SWNT as these two classes are not chiral and therefore no eMChA should be observable in such tubes. On the basis of 

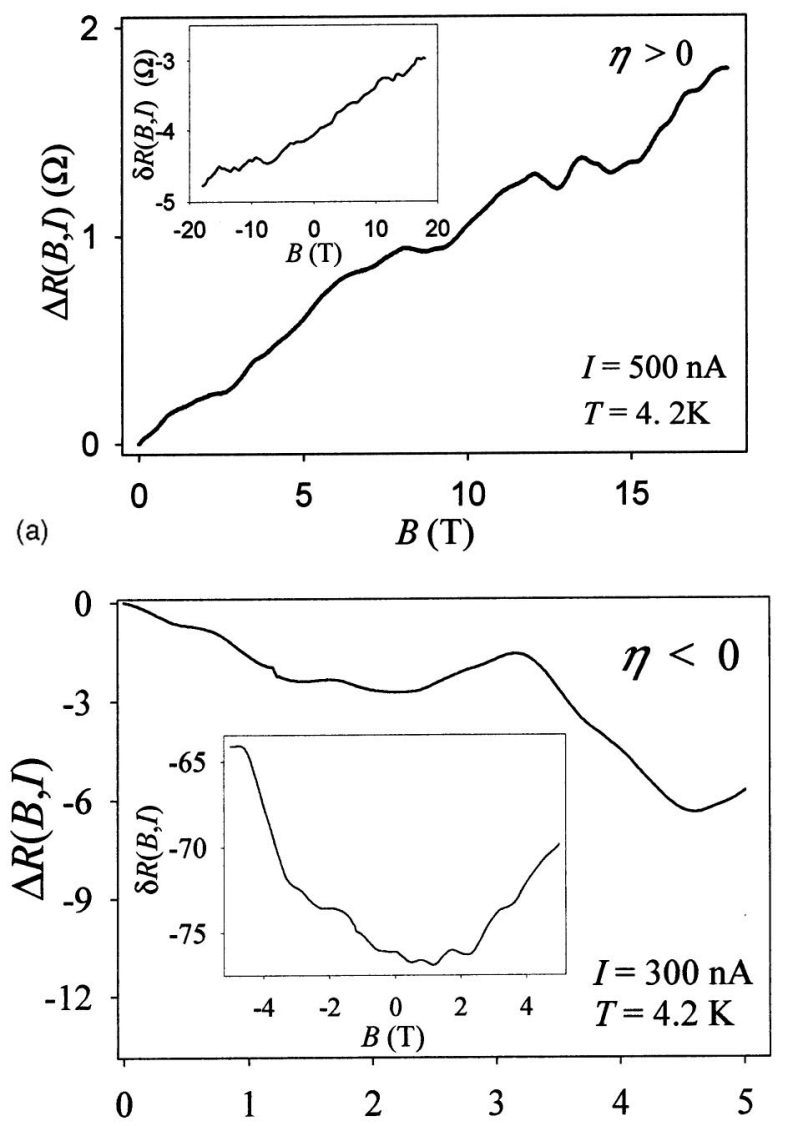

(b)

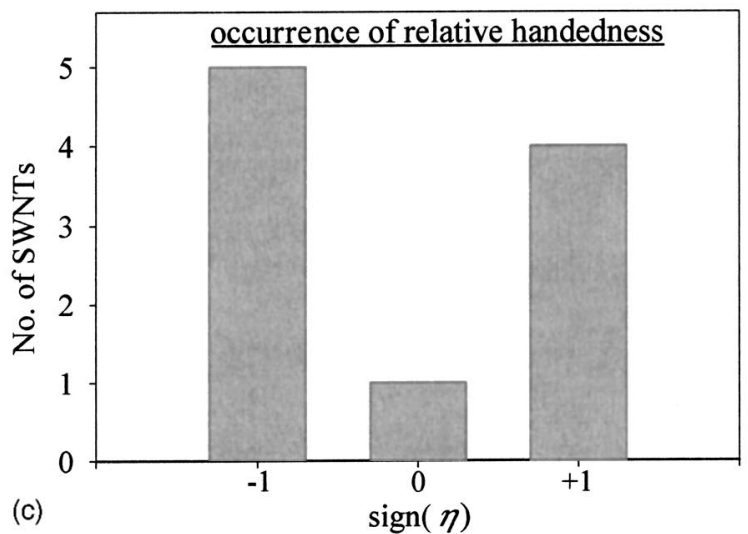

FIG. 2. (a) $\Delta R(B, I)$ vs $B$ of the SWNT sample in Fig. 1 at $I=500 \mathrm{nA}$ and $T=4.2 \mathrm{~K}$. In the inset $\delta R(B, I)$ vs $B$ is shown. The linear term in the eMChA determines the shape of the curve and only small contributions from higher order terms are present. (b) Inset $\delta R(B, I)$ vs $B$ for another SWNT at $I=300 \mathrm{nA}$ and $T=4.2 \mathrm{~K}$. The result is asymmetric in $B$ but dominated by contributions quadratic in $B$. Main figure: $\Delta R(B, I)$ as a function of $B$. From the plot $\eta<0$ can be deduced, i.e., this tube must be of opposite handedness as the tube in (a). (c) Histogram showing the occurrence of the relative handedness in all investigated samples.

AFM data, we can estimate that the diameters of all the investigated SWNTs are between 1.0 and $1.4 \mathrm{~nm}$, for which 50 possible $(n, m)$ pairs can be found. Among these are 3 armchair and 5 zig-zag nanotubes, of which 3 and 1, respectively, are metallic. Out of the chiral nanotubes 12 are metallic, but as they are doubly-degenerate due to their chirality,

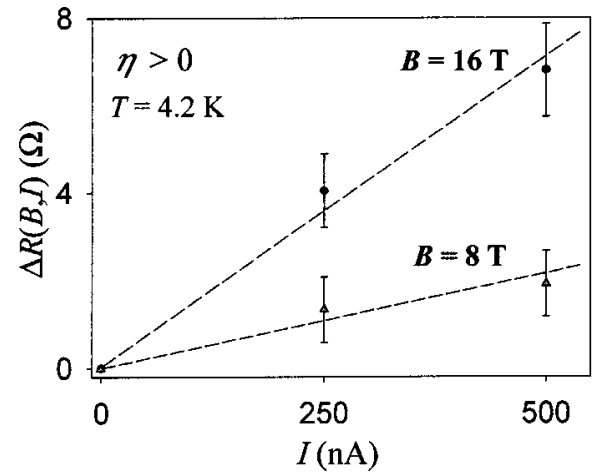

FIG. 3. Current dependence of the eMChA. $\Delta R(B, I)$ is shown as a function of $I$ for two different magnetic field strengths at liquid helium temperature (dotted lines are guides to the eye).

we have 24 chiral metallic tube types. So when investigating metallic SWNT in this diameter range, we should expect that 28 types are metallic, of which 4 are achiral. Therefore, under the assumption that no chiral preference has existed during nanotube synthesis, one should expect that of the metallic tubes, $43 \%$ is left-handed, $43 \%$ is right-handed, and only $14 \%$ is achiral. This is in good agreement with our assignment that the experimentally observed sign of $\eta$ corresponds to the handedness of the tubes.

Equation (1) predicts the eMChA to be proportional to the current traversing the nanotube. In Fig. $3 \Delta R(B, I)$ is plotted against the current at 16 and $8 \mathrm{~T}$ for a SWNT with $\eta>0$. A linear dependence is observed, in good agreement with Eq. (1).

Figure 4 shows a typical temperature dependence of $\Delta R(B, I)$. In general, we have not observed any eMChA in a SWNT above $50 \mathrm{~K}$, and in the range $4.2-1.5 \mathrm{~K}$, no significant temperature dependence of $\Delta R(B, I)$ is observed. We attribute the strong increase of $\Delta R(B, I)$ for $T \leqslant 4.2 \mathrm{~K}$ not to the substrate temperature itself, but to the increased cooling of the tube when it is immersed in liquid helium. This would imply that for higher substrate temperatures, when cooling occurs by helium gas, the tube lattice temperature or the

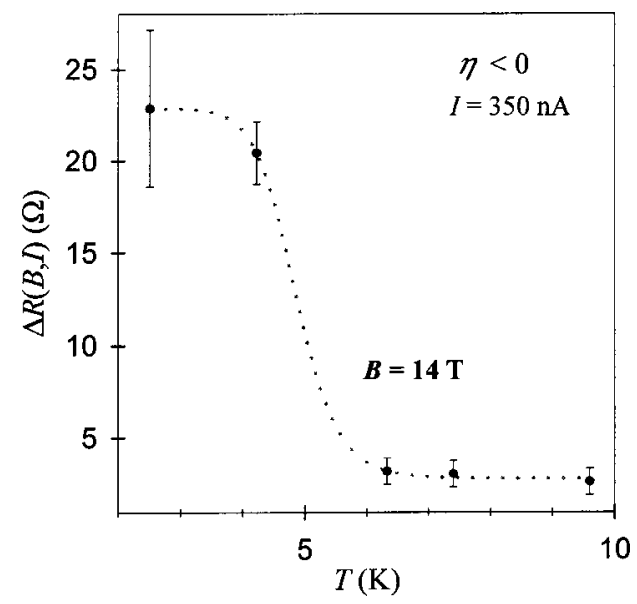

FIG. 4. Temperature dependence of the eMChA. $\Delta R(B, I)$ is plotted as a function of temperature at $B=14 \mathrm{~T}$ and $I=350 \mathrm{nA}$. Temperatures above 4.2 $\mathrm{K}$ were obtained by gas cooling, the others by liquid helium cooling. The dotted line is a guide to the eye. 
electron temperature is significantly higher than the substrate temperature due to heating by the high current densities, and that the true dependence of $\Delta R(B, I)$ on the electron temperature may be much weaker than that suggested by Fig. 4. Note that the increase of $\Delta R(B, I)$ with decreasing temperature shows that eMChA is not some hot-electron effect, as one might associate with a current dependent resistance, but an intrinsic contribution of chirality to magnetotransport. It would be interesting to determine the angular dependence of the eMChA, which Eqs. (2) and (3) predict to be of a $\cos \theta$ type ( $\theta$ is the angle between current and field). We did not succeed so far, probably because the large Lorentz force that is inherent to the large current densities and a perpendicular field component, irreversibly changes the samples. However regardless of the outcome of such an experiment, the fundamental symmetry considerations presented here that lead to Eq. (3) do not leave any room for other interpretations of the data obtained at parallel field than eMChA.

The microscopic origin of the observed eMChA can be of various types, e.g., scattering of charge carriers by chiral phonons ${ }^{16}$ or the magnetic self-field effect. However, the magnetic self-field effect which in combination with the normal quadratic magnetoresistance yields a chiral resistance contribution linear in $B$ and $j,{ }^{21}$ is estimated to be smaller than $5 \mathrm{k} \Omega / A T$ for $S W N T s$, which is too small to explain the observed effects. Electron-phonon scattering is ruled out in view of the observed temperature dependence. Thus, remaining explanations of the eMChA are scattering by chiral objects, like electron-defect or electron-electron scattering. ${ }^{21}$ Noteworthy, it has been suggested that a low temperatures, electron-electron correlations become important to electronic transport. ${ }^{10}$ An interpretation of the observed eMChA in terms of chiral electron-electron scattering would be consistent with this suggestion. Alternatively, the electronelectron correlation may reduce screening, so that electrondefect scattering becomes more important. In both cases, increasing eMChA with decreasing temperature is to be expected.

The simplest microscopic model to theoretically describe the eMChA for a SWNT is the case of an electron confined to a helix with a pitch $p .{ }^{24}$ Treating this model in the frame of quantum mechanics shows that contributions of odd power in $j$ and $B$ are present in the conductivity. ${ }^{25}$ The model assumes diffusive electronic transport along the helix with achiral scatterers. Neglecting all electron-electron interaction effects other than those leading to mass renormalization and scattering leads to the Hamiltonian, ${ }^{24,25}$

$$
\begin{aligned}
H= & -\frac{\hbar^{2}}{2 m\left(a^{2}+b^{2}\right)} \frac{\partial^{2}}{\partial \varphi^{2}}-i \hbar \frac{q}{2 m} \frac{a^{2}}{a^{2}+b^{2}} \\
& \times B \frac{\partial}{\partial \varphi}+\frac{q^{2} a^{2}}{8 m} B^{2}
\end{aligned}
$$

with electron mass $m, N$ the number of turns of the helix, the pitch $p=(2 \pi b)^{-1}$ and in the presence of a static magnetic field $B \hat{z}$. The energy-eigenvalues of the Hamiltonian are given by ${ }^{25}$

$$
E_{n}=\frac{\hbar^{2} n^{2}}{2 m N^{2}\left(a^{2}+b^{2}\right)}+\frac{q n \hbar}{2 m N} \frac{a^{2}}{a^{2}+b^{2}} B+\frac{q^{2} a^{2}}{8 m} B^{2},
$$

where three contributions are found: a zero-field energy

$$
E_{\mathrm{zf}, n} \equiv \frac{\hbar^{2} n^{2}}{2 m N^{2}\left(a^{2}+b^{2}\right)},
$$

a diamagnetic energy,

$$
E_{\mathrm{dia}} \equiv \frac{q^{2} a^{2}}{8 m} B^{2}
$$

and a magneto-chiral anisotropy energy

$$
E_{\mathrm{eMChA}, n} \equiv \frac{q n \hbar}{2 m N} \frac{a^{2}}{a^{2}+b^{2}} B=\frac{\pi N}{2} a^{2} j_{z, n} B,
$$

where

$$
j_{z, n}=\frac{q \hbar n}{\pi m N^{2}\left(a^{2}+b^{2}\right)}
$$

is the current in the $\hat{z}$ direction carried by the state characterized by the quantum number $n .^{25}$ Using a constant relaxation time approximation ${ }^{15}$ and taking into account the restricted motion of the electron on the helix the longitudinal conductivity reads 25

$$
\begin{aligned}
\sigma\left(j_{z}, B\right) \approx & \frac{q^{2} \tau}{k_{B} T} \frac{2 b^{2}\left|n_{F}\right|}{m\left(a^{2}+b^{2}\right)}\left(1+\frac{E_{\mathrm{eMChA}, n}}{N E_{\mathrm{zf}, n_{F}}}\right. \\
& \left.+\frac{a^{2} E_{\mathrm{dia}}}{\left(a^{2}+b^{2}\right) E_{\mathrm{zf}, n_{F}}}\right)\left(1-\frac{E_{\mathrm{eMChA}, n_{F}}}{2 E_{\mathrm{zf}, n_{F}}}\right)
\end{aligned}
$$

leading in consideration of the dominating terms to an eMChA contribution,

$$
\begin{aligned}
\xi_{\mathrm{eMChA}} \equiv \frac{\sigma\left(j_{z}, B\right)-\sigma\left(-j_{z}, B\right)}{\sigma\left(j_{z}, B\right)+\sigma\left(-j_{z}, B\right)} & \approx \frac{E_{\mathrm{eMChA}, n_{F}}}{2 E_{\mathrm{zf}, n_{F}}} \\
& \approx \frac{\pi N a^{2} j_{z, n_{F}} B}{4 E_{\mathrm{zf}, n_{F}}} .
\end{aligned}
$$

Using typical parameters of the SWNTs experimentally investigated above, that is, $a=0.6 \mathrm{~nm}, 2 \pi b=1.5 \mathrm{~nm}$, and $N=120$ for a corresponding helix of this size, and a Fermi energy $E_{F}=1.6 \cdot 10^{-19} \mathrm{~J}$ resulting in $n_{F}=400$, Eq. (7) yields the value $\xi_{\mathrm{eMChA}} / B \approx 8 \cdot 10^{-5} \mathrm{~T}^{-1}$ at a current of $0.8 \mu \mathrm{A}$. Comparison of the predicted value of $\xi_{\mathrm{eMChA}} / B$ and the experimental value $\xi_{\text {exp }} / B \approx 10^{-4} \mathrm{~T}^{-1}$ at a current of $j$ $=0.3 \mu \mathrm{A}$ shows a reasonable agreement.

In the present work we have reported for the first time the observation of electrical magneto-chiral anisotropy in molecular conductors. The main features of the effect, the dependence on magnetic field and current have been confirmed and the magnitude of the observed eMCHA is in good agreement with a theoretical prediction based on a helixmodel. Opposite effects in sign have been found with similar abundances and a SWNT with no eMChA has been observed which may be identified as an achiral type. The relative frequencies of the occurrence of these different types is in agreement with statistical predictions, assuming that there is 
no enantioselection during the SWNT production process. The observation of eMChA proves that the current through the SWNT has a chiral component in contrast to the isotropic nature of the in-plane conductivity of graphite. ${ }^{15}$

The authors gratefully acknowledge G. Wagnière, B. van Tiggelen, G. Martinez, P. Wyder, and A.G.M. Jansen for critical reading of the manuscript and stimulating discussions. C. Strohm is thanked for experimental support. The Grenoble High Magnetic Field Laboratory is a "laboratoire conventionné aux universités UJF et INP de Grenoble."

${ }^{1}$ S. Iijima, Nature (London) 56, 354 (1991).

${ }^{2}$ J. W. G. Wildöer, V. C. Lisbeth, A. G. Rinzler, R. E. Smalley, and C. Dekker, Nature (London) 391, 59 (1998).

${ }^{3}$ T. M. Odem, J. L. Huang, and C. M. Lieber, Nature (London) 391, 62 (1998).

${ }^{4}$ R. A. Jishi, D. Inomata, K. Nakao, M. S. Dresselhaus, and G. Dresselhaus, J. Photogr. Sci. 63, 2252 (1994).

${ }^{5}$ R. Saito, G. Dresselhau, and M. S. Dresselhaus, Physical Properties of Carbon Nanotubes (Imperial College Press, London, 1998).

${ }^{6}$ A. Joria, R. Saito, C. M. Lieber, M. Hunter, T. McClure, G. Dresselhaus, and M. S. Dresselhaus, Phys. Rev. Lett. 86, 1118 (2001).

${ }^{7}$ W. Clauss, M. Freitag, D. J. Bergeron, and A. T. Johnson, in Electronic Properties of Novel Materials: Science and Technology of Molecular nanostructures, XIII International Winterschool, Kirchberg 1999, AIP Conference Proceedings 486, edited by H. Kuzmany, J. Fink, M. Mehring, and S. Roth (AIP, New York, 1999), pp. 308-312.
${ }^{8}$ D. H. Cobden, M. Bockrath, P. L. McEuen, A. G. Rinzler, and R. E. Smalley, Phys. Rev. Lett. 81, 681 (1996).

${ }^{9}$ M. Bockrath, D. H. Cobden, P. L. McEuen, N. G. Chopra, A. Zettel, A. Thess, and R. E. Smalley, Science 275, 1922 (1997).

${ }^{10}$ M. Bockrath, D. H. Cobden, J. Lu, A. G. Rinzler, R. E. Smalley, L. Balents, and P. L. McEuen, Nature (London) 397, 598 (1999).

${ }^{11}$ H. R. Shea, R. Martel, and P. Avouris, Phys. Rev. Lett. 84, 4441 (2000).

${ }^{12}$ A. Bachtold, C. Strunk, J. P. Salvetat, J. M. Bonard, L. Forró, T. Nussbaumer, and C. Schönenberger, Nature (London) 397, 673 (1999).

${ }^{13}$ S. Frank, P. Poncharal, Z. L. Wang, and W. de Heer, Science 280, 1744 (1998).

${ }^{14}$ V. Krstić, S. Roth, and M. Burghard, Phys. Rev. B 62, R16353 (2000).

${ }^{15}$ Y. Miyamoto, S. G. Louie, and M. L. Cohen, Phys. Rev. Lett. 76, 2121 (1996).

${ }^{16}$ O. V. Kibis, Phys. Solid State 43, 2336 (2001).

${ }^{17}$ E. V. Ivchenko and B. Spivak, cond-mat/0202286v1, 2002.

${ }^{18}$ G. L. J. A. Rikken and E. Raupach, Nature (London) 390, 493 (1997).

${ }^{19}$ G. L. J. A. Rikken and E. Raupach, Phys. Rev. E 58, 5081 (1998).

${ }^{20}$ L. D. Landau and E. M. Lifshitz, Statistical Physics, Part 1, 3rd ed. (Pergamon, Oxford, 1980), p. 359 ff.

${ }^{21}$ G. L. J. A. Rikken, J. Fölling, and P. Wyder, Phys. Rev. Lett. 87, 236602 (2001)

${ }^{22}$ V. Krstić, G. S. Duesberg, J. Muster, M. Burghard, and S. Roth, Chem. Mater. 10, 2338 (1998).

${ }^{23}$ The electrical conduction, and thereby the eMChA is attributed to one individual nanotube in the bundle, which typically contains a few tubes, since the best contacted SWNT in the bundle will dominate the transport.

${ }^{24}$ I. Tinoco and R. W. Woody, J. Chem. Phys. 40, 160 (1964).

${ }^{25}$ V. Krstić and G. L. J. A. Rikken, Chem. Phys. Lett. 364, 51 (2002). 